UDC 621.315

\title{
Electron backscatter diffraction in the silicon nanowires
}

\author{
K.K. Dikhanbayev ${ }^{1, *}$, V.A. Sivakov², F. Talkenberg ${ }^{2}$, G.K. Mussabek ${ }^{1}$, \\ Ye.T. Taurbayev ${ }^{1}$, N.N. Tanatov ${ }^{1}$, E. Shabdan ${ }^{1}$ \\ ${ }^{1}$ Al-Farabi Kazakh National University, IETP, al-Farabi 71, 050040 Almaty, Kazakhstan \\ ${ }^{2}$ Leibniz Institute of Photonic Technology, Technology e.V. PF 100239, 07702 Jena, Germany \\ 'e-mail:dkadyrjan@mail.ru
}

\begin{abstract}
In this paper we consider the formation on the surface of silicon by metal-induced chemical etching, the silicon nanowires and the study of their electron (SEM) and (TEM) microscopy, X-ray diffraction (EDX) analysis and electron backscatter diffraction (EBDS) in nanowires. Combination of field emission SEM and EBSD possible to determine the orientation of the individual grains, the local texture oriented correlation on solid surfaces of polycrystalline material. This method of producing silicon nanowires has a number of the above-mentioned advantages over other methods. In addition, the studied objects themselves exhibit interesting optical properties, such as the localization of light, photoluminescence (PL), very low reflectance $(<10 \%$ at $300-800$ $\mathrm{nm})[1]$ and high absorption [2] (>90\% at $500 \mathrm{~nm})$.
\end{abstract}

Key words: silicon, silicon nanowires, morphology, metal-induced chemical etching, electron backscatter diffraction. PACS numbers: 81.07.-b, 61.46.Np

\section{Introduction}

It is now one of the fastest growing areas in physics is the study of the fundamental properties of solid-state nanostructures and nanomaterials produced using the latest nanotechnology [3]. This work is devoted to the study of nanostructures of silicon obtained by chemical and electrochemical etching of single crystal silicon, as well as exploring the possibilities of using such properties in photovoltaic applications [4].

Very interesting material for research from this perspective proved silicon nanostructuring modifications which have different morphologies thanks to its unique properties can be used in key areas of life of modern people, such as nanoelectronics, photonics, energy and biomedicine. [5]

In this paper considered the technology of producing silicon nanowires using metal induced by chemical etching of silicon wafers. The content of this method is that the surface of the crystalline silicon precoated with silver, followed by a redox reaction, and then etched in a solution comprising HF: $\mathrm{H}_{2} \mathrm{O}_{2}$.

Metal-stimulated etching is appealing for researchers because it is cost-effective and simple method of forming silicon nanostructures with the possibility of controlling their parameters (such as length, diameter, orientation, type and level of doping) [6].
In the current study, monocrystalline silicon nanowire arrays (SiNWs) were prepared through a metal-assisted wet chemical etching method of silicon wafers in an etching solution composed of HF and $\mathrm{H}_{2} \mathrm{O}_{2}$. Morphology properties of the monocrystalline SiNWs are improved greatly with the formation of the nanostructure on the silicon wafers. By controlling the hydrogen peroxide concentration in the etching solution, SiNWs with different morphologies and surface characteristics are obtained.

Subsequently, the silicon surfaces were immersed into the first solution (thermostateted by $920^{\circ} \mathrm{C}$ ) containing $5 \mathrm{M} \mathrm{HF}$ and $0.01 \mathrm{M} \mathrm{AgNO}_{3}$ [7]. The effect of silver nanoparticles growth on different crystallographic oriented silicon wafers was investigated.

\section{Experiment}

The crystalline of the silver nanostructures on the silicon surfaces was investigated in cross-section view using coupling of scanning electron microscopy and electron backscatter diffraction. The SEM+EBSD pattern results are presented in Fig. 1. The crystallography studies based on EBSD and stereographic pole-figure on the surfaces covered with silver nanostructures present the polycrystalline nature of the silver nanoparticles deposited in the first step of the electro-less etching of silicon surfaces. 


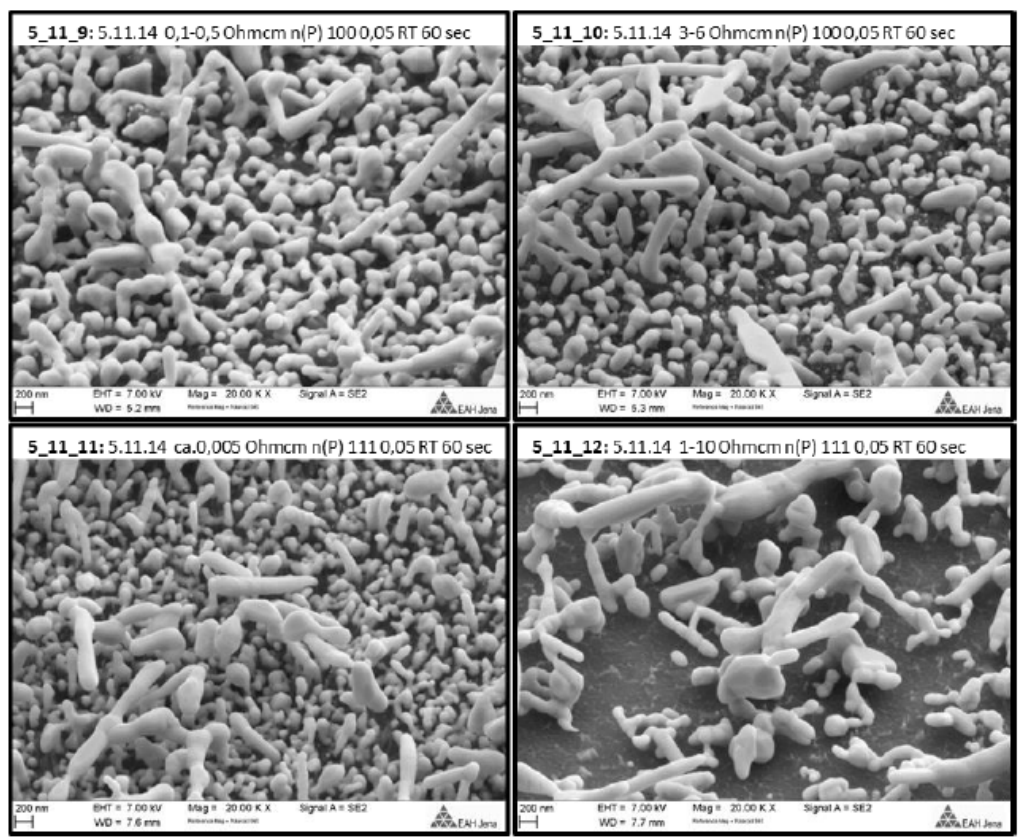

Figure 1 - Scanning electron microscopy micrographs

of silver nanostructures deposited on silicon surfaces with different doping level.

The results are presented in Fig 1. Structural analysis of the silver nanostructures was carried out by field emission scanning electron microscopy (Carl Zeiss ULTRA 55).

On the left side the silver nanostructures grown on the $<100>$ and on the right side $<111>$-oriented wafers are displayed. It is clearly visible that the orientation of the silicon substrate does not affect the morphology of the silver deposition on silicon. In Fig 2, the deposits are shown for $\mathrm{n}$-doped silicon with an orientation of $<100>$ and $<111>$. In the right column, the deposits with a low doped substrate are displayed, in the left column, the nanostructures with a highly doped substrate are presented.
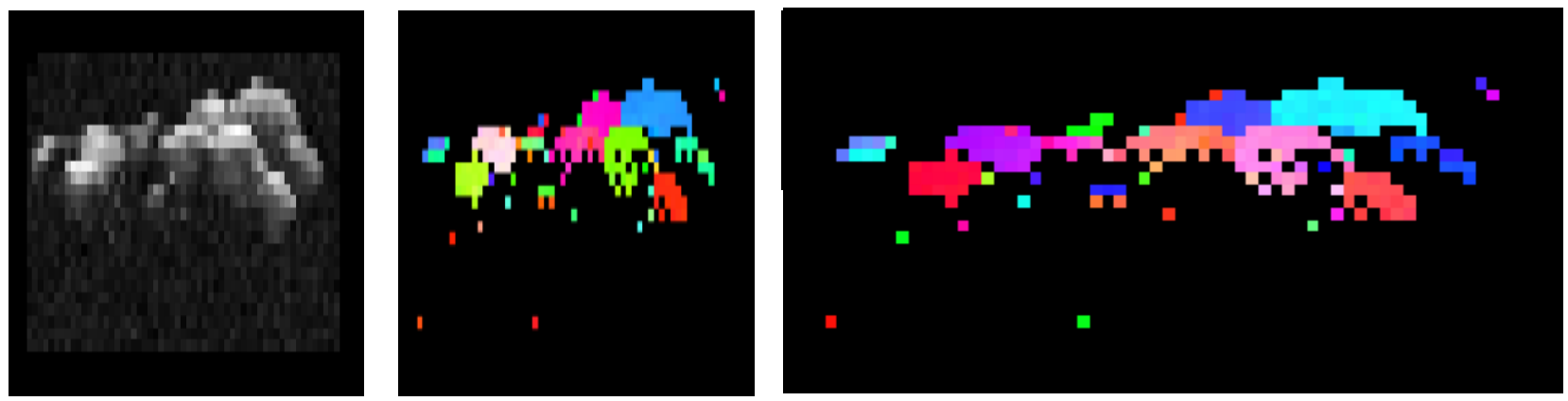

Figure 2 - SEM and EBSD pattern on silver nanostructures deposited from $\mathrm{AgNO}_{3} / \mathrm{HF}$ solution on $\mathrm{Si}(100)$ surface.

After a uniform layer of $\mathrm{Ag}$ nanoparticles was coated, the wafers were then immersed in the etchant solution composed of $\mathrm{HF}, \mathrm{H}_{2} \mathrm{O}_{2}$, and $\mathrm{H}_{2} \mathrm{O}$ (the volume ratios $\mathrm{HF} / \mathrm{H}_{2} \mathrm{O}_{2}: 1: 10,1: 20,1: 30,1: 40$ ) at room temperature in a sealed Teflon vessel. The $\mathrm{Si}$ wafers were immersed in a solution of concentrated nitric acid solution to remove the excess $\mathrm{Ag}$ nanoparticles, rinsed with deionized water, and then dried in vacuum at $60^{\circ} \mathrm{C}$. Structural analysis of the silicon nanostructures was carried out by field emission scanning electron microscopy (FEI Helios NanoLab FESEM). 
The dependence is nonlinear and it can be fitted by the following expression:

$$
\mathrm{L}=\mathrm{Ct}^{\alpha}
$$

where, $\mathrm{L}$ the SiNWs length, $\mathrm{t}$ is the MAWCE time, and $\mathrm{C}$ and $\alpha$ are constant coefficients. The coefficient $\alpha$ was calculated from the data presented in Fig 3 and it is accounted 0.75.

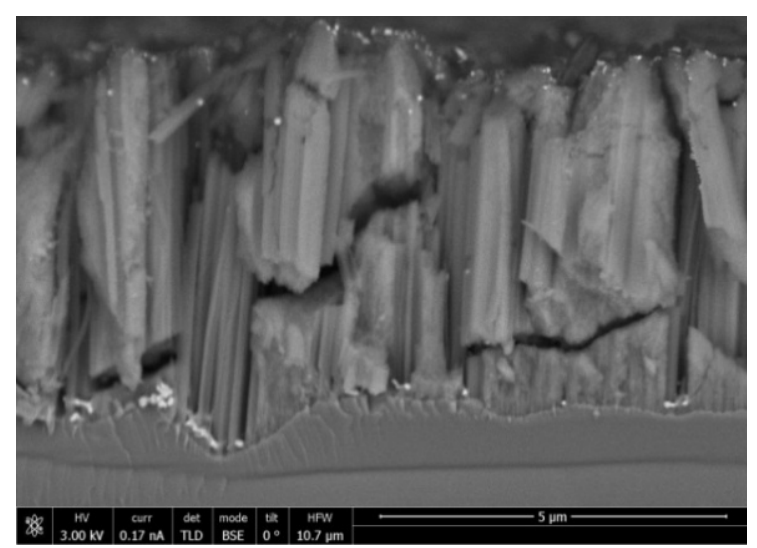

a

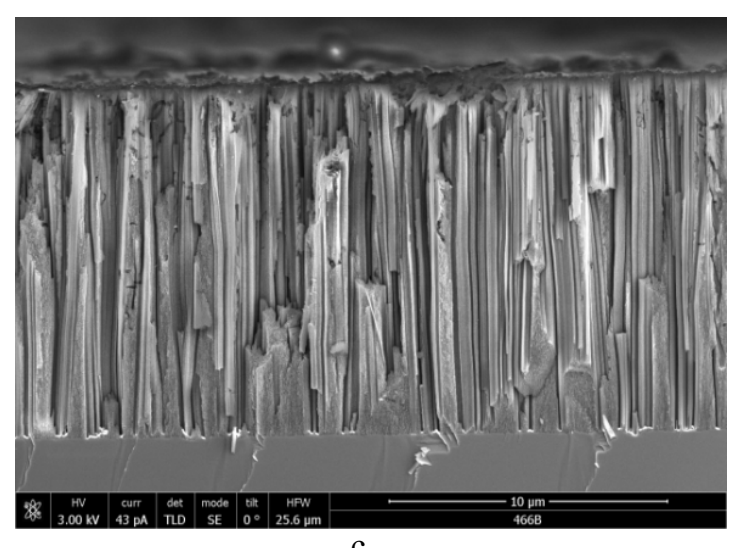

C
Scanning electron microscopy images of the asgrown silicon nanowires from the phosphorous doped n-Si (100) wafers with different concentrations of $\mathrm{H}_{2} \mathrm{O}_{2}$ are summarized in Fig 3. For 1:40, lowly porous nanowires were obtained as shown in SEM images Fig 3d. With increasing the concentration of $\mathrm{H}_{2} \mathrm{O}_{2}$, the silicon nanowires first become increasingly rough on the surface, then start to evolve porous shells surrounding the solid cores, and eventually form entirely porous nanowires.
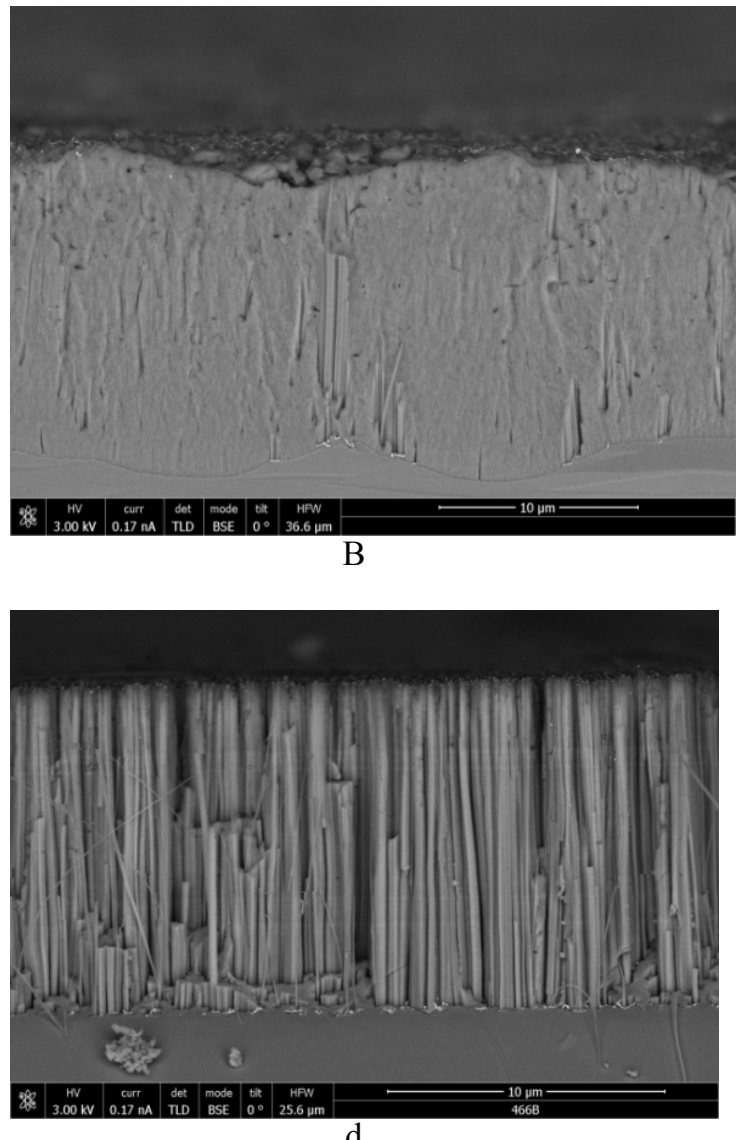

d

Figure 3 - SEM cross-sectional views of silicon (100)-oriented silicon wafers after metal-assisted etching using:

a) $1: 10 \mathrm{H}_{2} \mathrm{O}_{2} / \mathrm{HF}$; b) $1: 20 \mathrm{H}_{2} \mathrm{O}_{2} / \mathrm{HF}$; c) $1: 30 \mathrm{H}_{2} \mathrm{O}_{2} / \mathrm{HF}$; d) $1: 40 \mathrm{H}_{2} \mathrm{O}_{2} / \mathrm{HF}$.

These studies clearly demonstrate that the increase portion of the nanowires becomes porous with the decrease of the $\mathrm{H}_{2} \mathrm{O}_{2}$ concentration. Our results show that the increase of hydrogen peroxide concentration can result in increasingly porosity of nanowires. Since $\mathrm{H}_{2} \mathrm{O}_{2}$ itself cannot etch the $\mathrm{Si}$ off, the formation of porous structures should by facilitated by the metal nanoparticles [8].

\section{Energy Dispersive X-Ray analysis}

The chemical composition of silicon nanostructures was investigated using Energy Dispersive X-Ray analysis coupled on Bruker EDX system. The typical EDX spectra of silicon nanowires after cleaning with $\mathrm{HNO}_{3}$ (remove of silver nanoparticles) is presented in Fig 4. 
From EDX spectrum,it is clearly visibly that the surface with silicon nanowires contains only elemental silicon and oxygen as an evidence of the presence of native oxide. The elements distribution was investigated in mapping EDX modus as shown in Fig 4. It was found that surface contains only silicon and oxygen.

It was found that surface contains only silicon and oxygen. It has also been discovered the long time EDX measurements on the etched silicon nanowires shown presence of the fluor and silver as residual elements.

The presence of silver can be explained as not completed dissolution of silver nanoparticles in nitric acid. The presence of fluor can be explained as a result of in situ flor passivation of silicon passivation during the etching process (the etchant solution contains hydrofluoric acid). This is also confirmed in article [9].

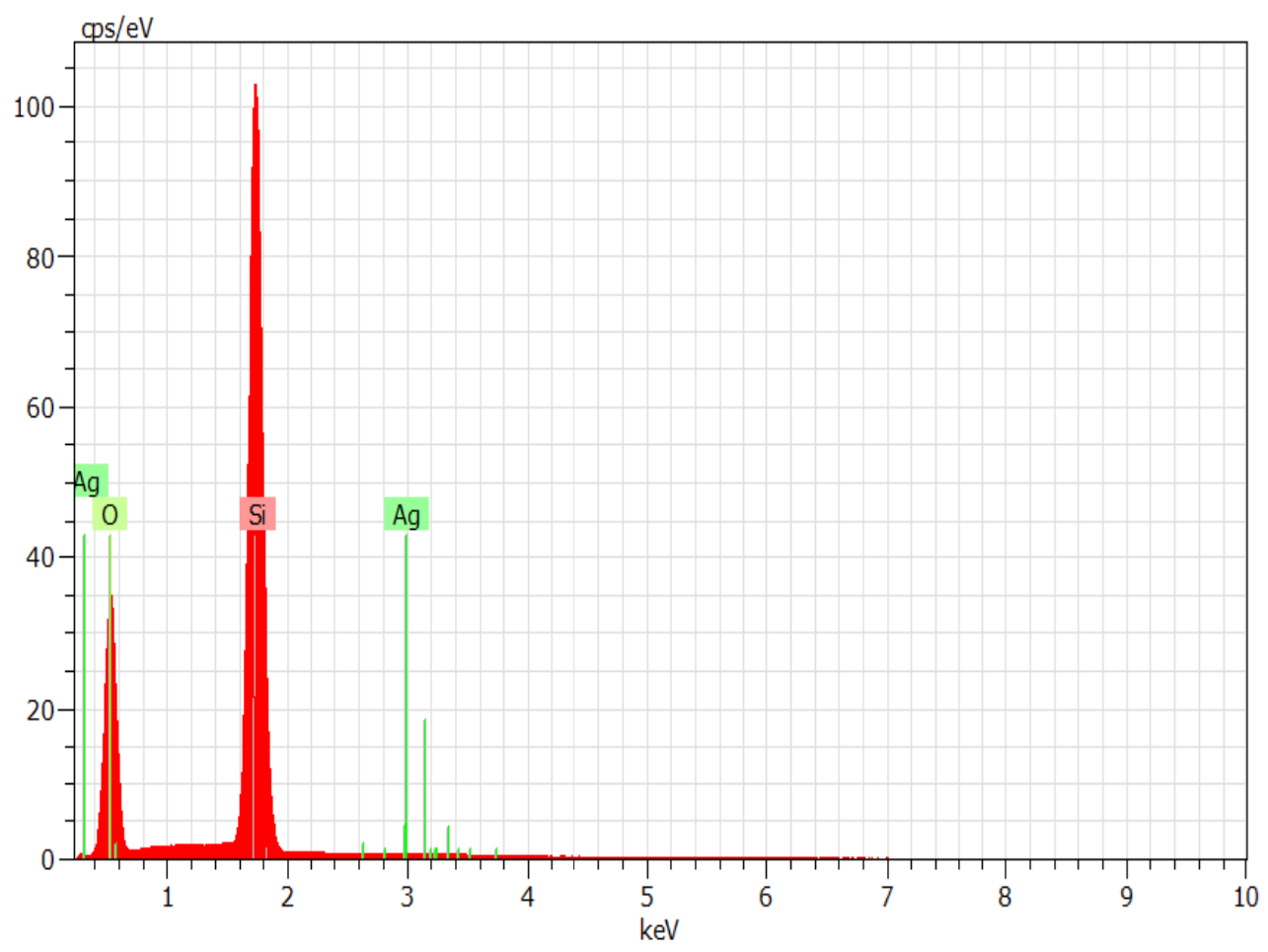

Figure 4 - Energy Dispersive X-Ray spectrum of Silicon nanowires formed form bulk silicon wafer using wet-chemical electroless etching.

\section{EBSD analysis}

The silicon wafer crystallographic orientation influence to the geometry and profile of silicon nanowires was studied by electron microscopy. Fig 5 a-b and Fig 5 c-d show the SEM cross sectional and single SiNW transmission electron microscopy (TEM) micrographs of the silicon nanowires etched from (100) and (111) monocrystalline silicon wafer, respectively.

Figure 5a shows straight SiNWs perpendicular to the wafer surface. The formation of SiNWs in Si(111) wafers appears to be more complicated (cf. Fig 5c), i.e. we can realize zig-zag SiNW orientations, reproducibly. We can exclude that plastic deformation is responsible for this morphology based on TEM investigations that show the SiNWs to be free of extended defects such as dislocations or twins or stacking faults at the very edge of a zig-zag SiNW as shown in TEM studies in Fig 5 d.

The growth direction and crystal structure of the silver nanostructures were investigated using Electron Backscatter Diffraction (EBSD) (Bruker eFlashHR EBSD Detector + ARGUSTM FSE/BSEDetector System).

Combining FE-SEM and EBSD allows the determination of individual grain orientations, local textures, and point to point orientation correlations on bulk surfaces of polycrystalline materials. EBSD studies are carried out on well separated SiNWs that were isolated on copper TEM grids as shown on Fig 6 . 
This is done by detaching the SiNWs from the silicon substrate using ultrasonic treatment in alcohol and dropping of the SiNW suspension onto the TEM grid where the dilute SiNWs reside after evaporation of the alcohol. EBSD in the SEM at good pattern quality is carried out using an acceleration voltage of $15 \mathrm{kV}$ and a probe current of approx. 2nA. These studies indicate that the etching process did not destroy the crystalline integrity of the structure.

Figure 7a shows straight SiNWs perpendicular to the wafer surface, as pointed out in previous papers.

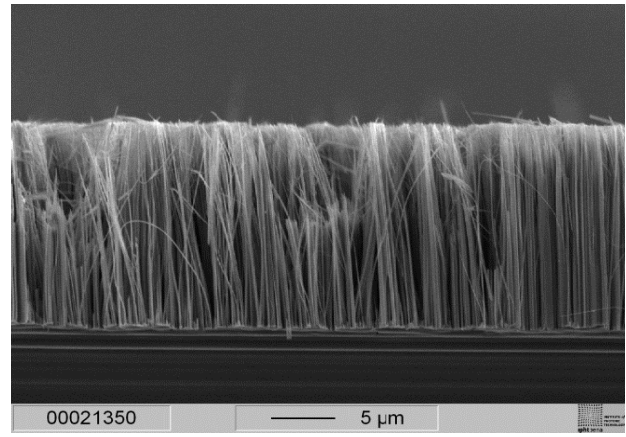

a

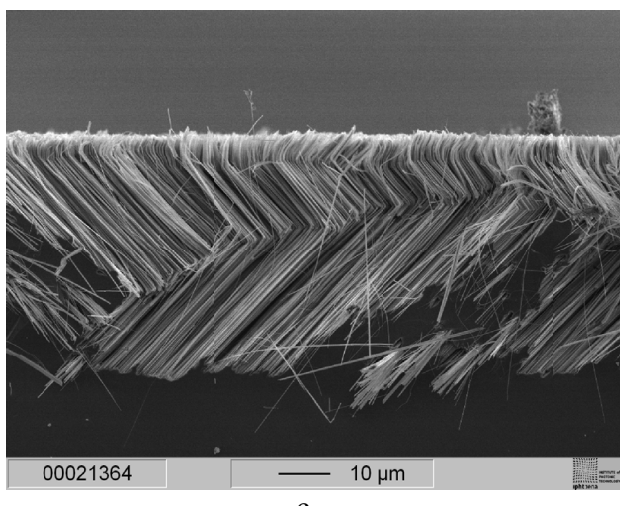

c
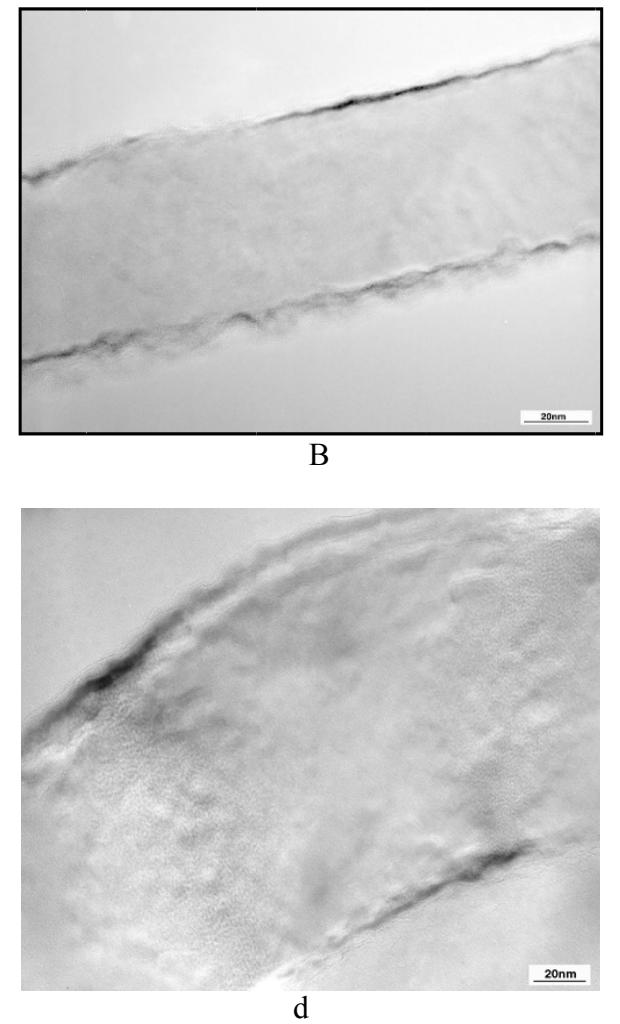

Figure 5 - a-b) SEM and TEM micrographs of SiNWs etched from single crystalline (100) silicon wafer; c-d) SEM and TEM micrographs of SINWs etched from single crystalline (111) silicon wafer.
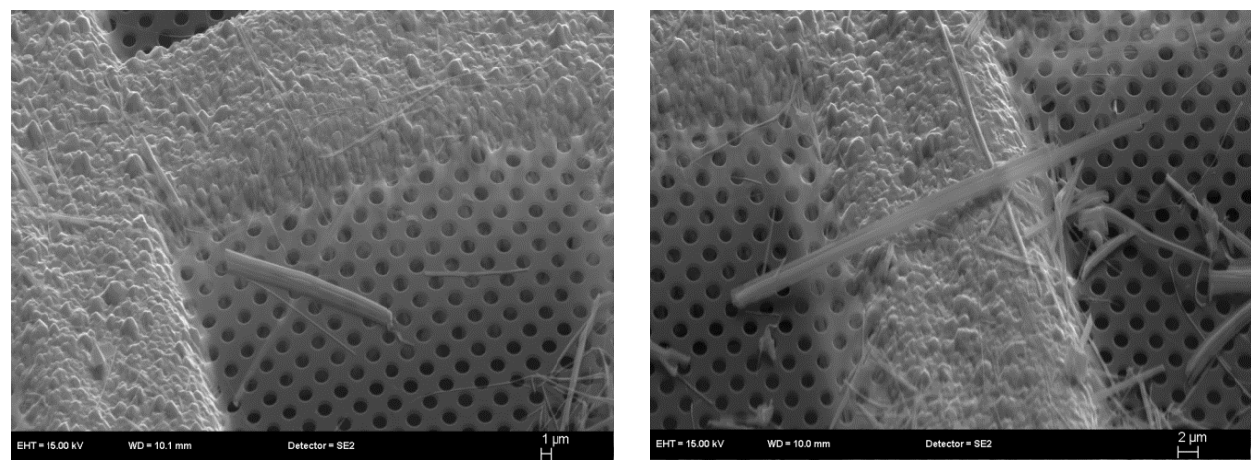

Figure 6-SEM micrographs of the silicon nanowires placed on copper TEM-grid for EDSD studies. 

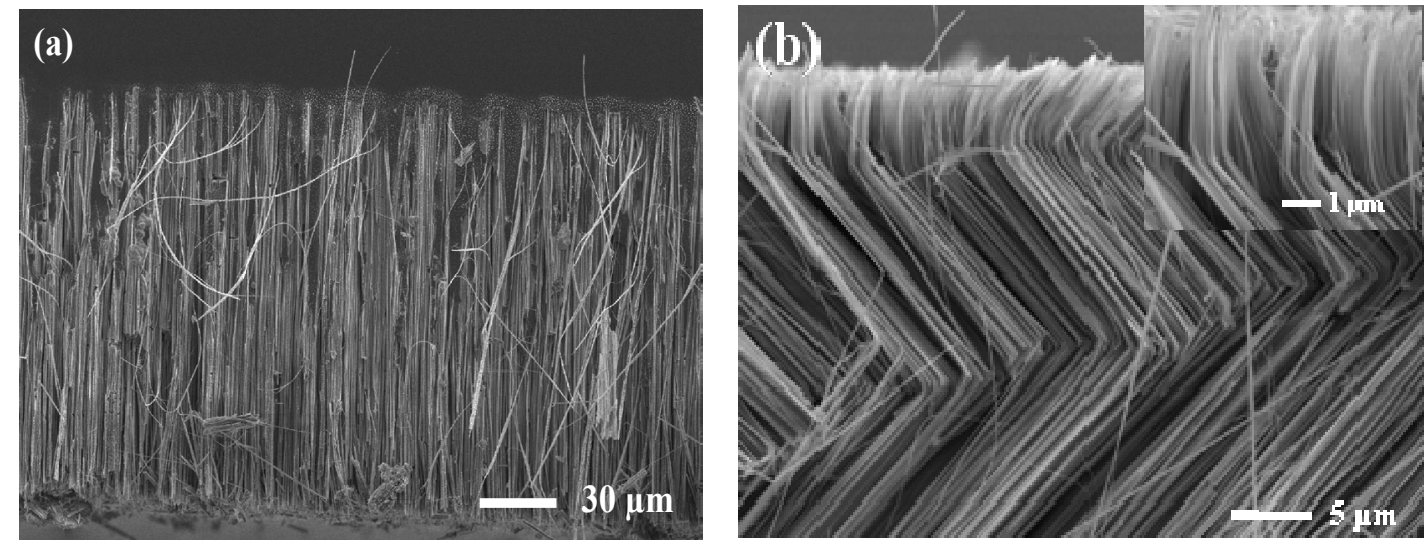

Figure 7 - Electron microscopy investigations to show the influence of the starting Si wafer orientation on the SiNW arrays architectures (a) p-Si(100) wafer $(0.7-1.5 \Omega \mathrm{cm})$ cross sectional SEM micrograph; (b) $\mathrm{p}$-Si(111) wafer (1-20 $\Omega \mathrm{cm})$ cross sectional SEM micrograph with inset of the zig-zag close to the wafer surface;

The formation of SiNWs in $\mathrm{Si}(111)$ wafers appears to be more complicated (cf. Fig. 7b), i.e. we can realize zig-zag SiNW orientations, reproducibly. We can exclude that plastic deformation is responsible for this morphology based on TEM investigations that show the SiNWs to be free of extended defects such as dislocations or twins or stacking faults at the very edge of a zig-zag SiNW.

We could show etching of $\mathrm{Si}(111)$ wafers for which we can realize straight (cf. Fig. 7a) etching along [111] directions or transition of [111] to [100] directions so that zig-zag (cf. Fig. 7b) architecture in
SiNWs forms. On this occasion, it was about the work [10].

To prove the varying etching directions we used electron backscatter diffraction (EBSD) in a SEM. Results of EBSD studies are shown in Fig. 8 and Fig. 9. Due to its excellent spatial resolution of a few $10 \mathrm{~nm}$ even over large probed areas, EBSD enables orientation analysis of SiNW with emphasis on grain size (or single crystallinity), grain distribution and SiNW orientations. Fig. 8 proves single crystallinity over the full SiNW length, as given by the single colour, coding the SiNW.

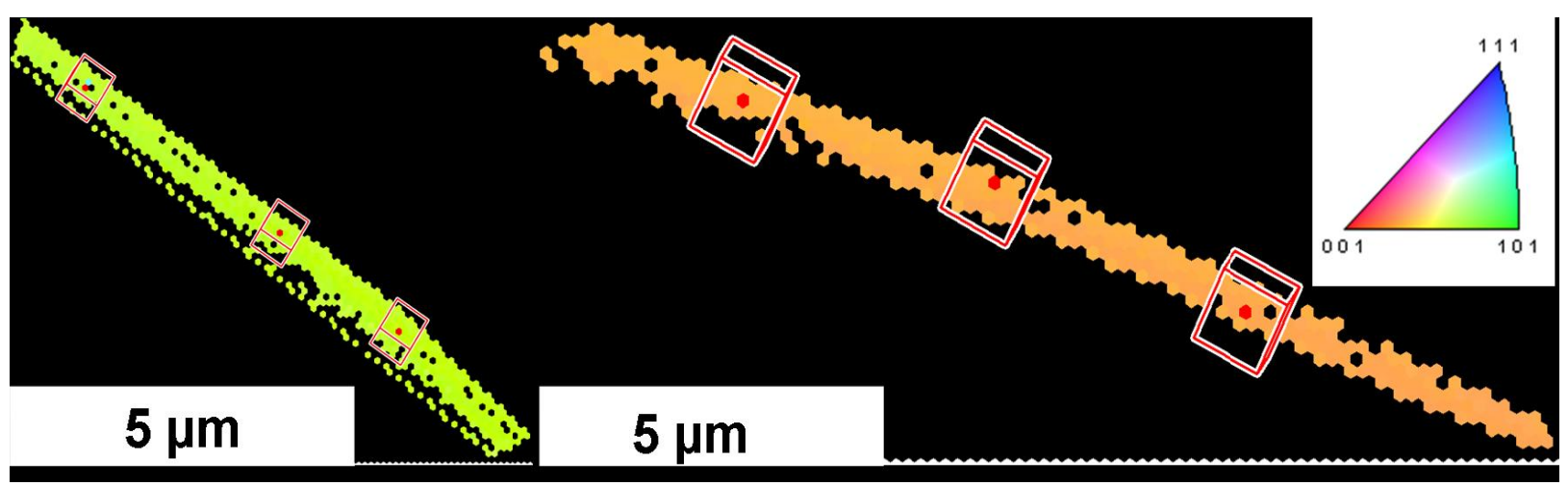

Figure 8 - EBSD analysis (inverse pole figure representation) of two silicon nanorods (SiNRs) fragments etched into a $\mathrm{Si}(111)$ wafer. The orientation cubes for both SiNWs show etching direction (parallel to the long axis of the SiNR) to be parallel to [100] direction. 

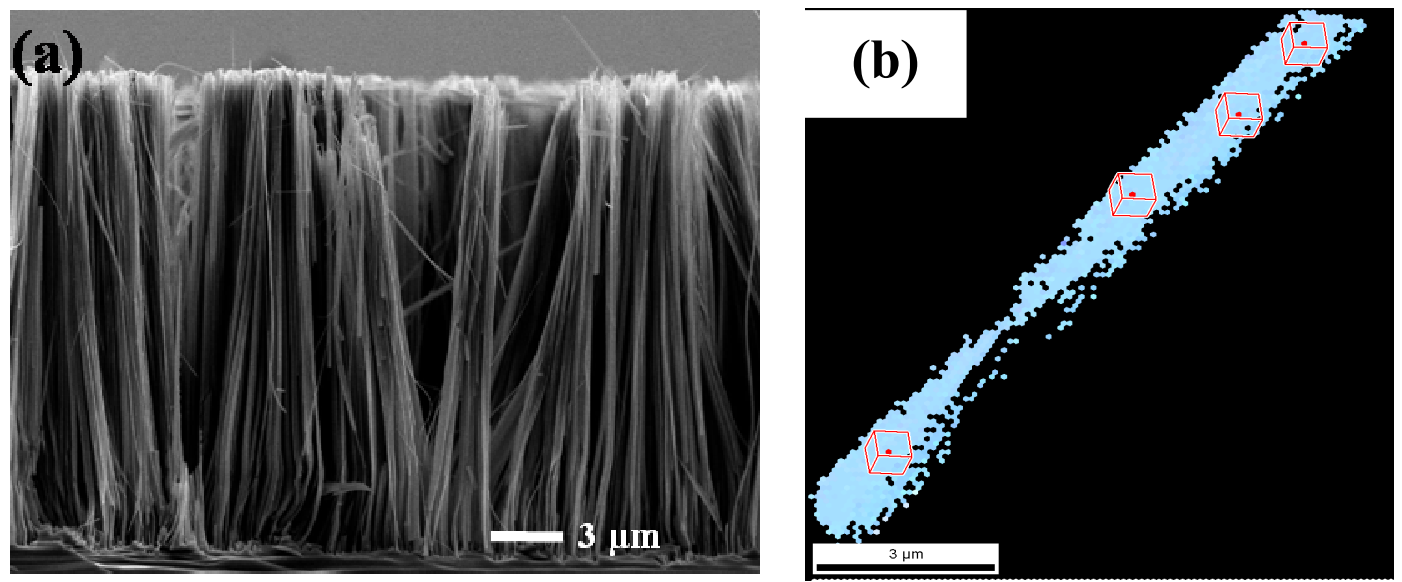

Figure 9 - (a) SEM micrograph of vertically etched SiNWs into a Si(111) wafer;

(b) EBSD analysis one SiNW fragment of such a wafer; colour coded inverse pole figure representation; the orientation cubes for this SiNW indicate etching direction (parallel to the long axis of the SiNW) to be parallel to the [111] direction.

The samples investigated in this work include: Sample A: Highly-doped etched Si wafers typically consisting of $\mathrm{N}_{\text {dope }} \approx 10^{20} \mathrm{~cm}^{-3}$, n-type c-Si (111), as dopant, 0.00015-0.005 ohm ·cm; Sample B: Weaklydoped etched $\mathrm{Si}$ wafers typically consisting of $\mathrm{N}_{\text {dope }}$ $\approx 10^{16} \mathrm{~cm}^{-3}$, p-type c-Si (111), as dopant, 5-10 $\mathrm{ohm} \cdot \mathrm{cm}$. The samples were un-passivized.

In contrast to the common use of pole figures for orientation representation in the following the present crystal orientation will be illustrated by orientation cubes showing the orientation of the unit cell with respect to the sample surface. Here every orientation cube belongs to an individual point of the EBSD scan and thus provides only information for the particular point.

According to that the determination of small deviations between different points could not be visualized and no statistic about the entire scan could. Nevertheless it provides a direct visualisation of the present crystal orientation and enables an intuitive access to the etching direction. Here it could be shown that etching SiNW from Si(100) wafer results in wires with a long axis almost parallel to the [100]-direction.

\section{Conclusion}

Structural analysis of the nanostructures of silver as measured by a scanning electron microscope with field emission showed that crystallographic orientation of the silicon substrate as in the $\langle 100\rangle$ and $\langle 111\rangle$ does not affect the morphology of the deposition of silver nanoparticles on its surface.
It was found that with increasing levels of silicon doping concentration increases the deposition of silver in both directions of orientation of silicon. Upon contact of the Schottky electrons diffuse from the silicon silver, thereby generating depletion layer where silicon is charged positively and negatively silver.

As a result, metal-induced wet chemical etching (MAWCE) it was found that with increasing hydrogen peroxide concentration in the etching agent leads to a high porosity nanowire. Increasing the concentration of hydrogen peroxide generates an increase in the number of free ions $\mathrm{Ag}+$, and an increase in the dopant concentration - to increase the number of weak defect centres in the silicon lattice. Both of these factors are important for the formation of additional ways to main etch etching directed vertically.

EBSD measurements showed that etching on substrates Si (100) leads to the formation of filaments with the long axis is almost parallel to the [100] direction normal to the surface different colour coding. In the case, of a vertical SiNW on the substrate Si (111) direction of the etching almost parallel to the (111) plane.

\section{Acknowledgments}

The authors thank professor V.Yu. Timoshenko for fruitful discussions. Preparation of samples for TEM, SEM investigations by A.S. Dzhunusbekov is gratefully acknowledged. The authors gratefully acknowledge the German Science Foundation (Leibniz Institute of Photonic Technology, Jena, Germany) 


\section{References}

[1] M.S. Gudiksen, L.J. Lauhon, J. Wang, D.C. Smith, C.M Lieber. Growth of nanowire superlattice structures for nanoscale photonics and electronic // J. Nature. - 2002. - Vol. 415. - P. 617-620.

[2] V. Sivakov, G. Andra, A. Gawlik, A. Berger, J. Plentz, F. Falk, S.H Christiansen. Silicon NanowireBased Solar Cells on Glass: Synthesis, Optical Properties, and Cell Parameters // Nano Letters. - 2009. - Vol. 9. - P. 1549-1554.

[3] Hui Fang, Xudong Li, Shuang Song, Ying Xu, Jing Zhu. Fabrication of slantingly-aligned silicon nanowire arrays for solar cell applications // J. Nanotechnology. -2008. - Vol. 19. - P. 255703.1255703.6.

[4] D.A.G. Bruggeman. Berechnung verschiedener physicakalisherkonstantenvon heterogen substanzen // Ann. Phys. (Leipzig). - 1935. - Vol. 24. - P. 634-664.

[5] S. Chan. Porous silicon multilayer structures: from interference filters to light emitting devices to biosensors // PhD thesis. New York: University of Rochester. $-2000 .-155$ p.

[6] B.M. Kayes, et al. Synthesis and Characterization of Silicon Nanorod Arrays for Solar Cell
Applications // Photovoltaic Energy Conversion IEEE 4th World Conference Record. - 2006. - Vol.1. - P. 221-224.

[7] E. Galopin. Metal-assisted chemical etching of silicon: Preparation of silicon nanowire arrays // Electrochem. Soc. Meeting Abstracts. - 2008. - P. 2.

[8] G.K. Mussabek, V.Yu. Timoshenko, K.K. Dikhanbayev, A.S. Dzhunusbekov, T.I. Taurbayev, V.E. Nikulin, Ye.T Taurbayev. Antireflections coatings for silicon solar cells formed by wet chemistry methods // KazNU Bulletin, physics series. - 2013.- №2(45). - P. 14-19.

[9] K. Tsujino, M. Matsumura. Morphology of nanoholes formed in silicon by wet etching in solutions containing $\mathrm{HF}$ and $\mathrm{H}_{2} \mathrm{O}_{2}$ at different concentrations using silver nanoparticles as catalysts // Electrochim. Acta. 2007. - Vol. 53. - №1. - P. 28-34.

[10] L.A. Osminkina, K.A. Gonchar, V.S. Marshov, K.V. Bunkov, D.V. Petrov, L.A. Golovan, F. Talkenberg, V.A. Sivakov, V.Yu Timoshenko. Optical properties of silicon nanowire arrays formed by metal-assisted chemical etching: evidences for light localization effect. // Nanoscale Research Letters. - 2012. - Vol.7. - P. 524530 . 